\title{
Early detection of micro-organisms development on stone monuments thanks to the stimulated infrared thermography and SVD
}

\author{
by Stéphanie Eyssautier-Chuine ${ }^{*}$, Kamel Mouhoubi* ${ }^{* *}$ Fany Reffuveille ${ }^{* *}$, Jean-Luc Bodnar ${ }^{* \star}$ \\ * Groupe d'Étude sur les Géomatériaux et les Environnements Naturels Anthropiques et Archéologiques EA \\ 3795 (GEGENAA) - SFR Condorcet FR CNRS 3417 - 2, Esplanade Roland Garros, University of Reims \\ Champagne-Ardenne (URCA), 51100 cedex, Reims, France.stephanie.eyssautier@univ-reims.fr \\ ** CATHERM, GRESPI EA 4694, University of Reims Champagne-Ardenne (URCA), Moulin de la Housse \\ BP 1039, 51687 Reims, France.kamel.mouhoubi@univ-reims.fr, jean-luc.bodnar@univ-reims.fr \\ *** Biomatériaux et Inflammation en Site Osseux EA 4691 (BIOS), U.F.R. Pharmacie, 51, rue Cognacq Jay, \\ University of Reims Champagne-Ardenne (URCA), 51095 Reims Cedex, France. fany.reffuveille@univ- \\ reims.fr
}

\begin{abstract}
Stimulated IRT test has been achieved in the aim at detecting early biofilms on the stones monument before greening. The biocolonisation is often removed because of the unsightly aspect that gives the greening and darkening of the monuments. The SVD post processing is usually applied to decrease the thermal artefacts at the surface of a work of art and to improve the detection of flaws inside it. Here the SVD use was to detect biofilms as surface artefacts thanks to the first EOFs which are dedicated to highlight the variations of energy at the surface induced by the biofilms.
\end{abstract}

\section{Introduction}

Natural stones have been used throughout ages to raise buildings, monuments and sculptures and the historical and cultural significance of many monuments calls to its preservation. Stone deterioration is the combination of environmental factors comprising climatic conditions (temperature, humidity) and anthropic activities (air pollution). That leads to physical, chemical weathering and biological development. The colonisation of stone by micro-organisms causes on first an unsightly effect by a fouling and a change of stone colour. Over time, the living of organisms induces undesired change of stone properties by a biochemical action made by the cell metabolism and a mechanical action by the growth of the vegetation. At the final state, the weakness of the stone leads to its breakdown. Colourimetry is a usual NDT which assesses the biodeterioration of the stone through its colour change [1-3] but the settlement of pioneer bacteria can not be tracked by this method because of the lack of colour. IRT as a NDT has been investigated to detect pioneer biofilms before the visible soiling of walls to limit the cleaning. For this purpose, experiment was set on a limestone called "Courville stone" which is used in major buildings in the surroundings of Paris. Stone samples have been exposed in an outdoor platform for their development of the natural colonisation by bacteria. Three of them have been collected after few months of exposure and compared to three non-colonised sterile stones within IRT. Considering SVD analysis has already proved its significant performance in the thermography of works of art $[4,5]$, this post processing of thermograms has been applied in order to emphase the early development of bacteria on the stone surface.

\section{Singular value decomposition (SVD)}

\subsection{Principle}

The analysis of structures with a pulsed thermal excitation can be limited to the detection of internal structural anomalies as an artefact of a remote technique. Alternative basis functions provide interesting means to decompose thermal response data. A singular value decomposition makes a set of orthogonal statistical modes by the strongest projection of data [6]. This treatment builds the best empirical model of the experimentation. SVD is a useful tool for signal treatment since it needn't calculate the sum of trigonometric or exponential functions as the traditional Fourier or Laplace transforms. The approach is to specify a SVD of a defined data matrix resulting from the photothermal experiment. The matrix includes $M$ lines and $N$ columns $(M>N)$ as $M$ parameter is a thermogram pixels number and $\mathrm{N}$ parameter is the thermogram number. The resultant matrix consists of gathering in every column the thermogram pixels values. Then SVD is applied according to the following formula:

$$
\mathrm{X}=\mathrm{U} \cdot \Sigma \cdot \mathrm{V}^{\top}
$$

$\mathrm{U}$ is an $\mathrm{M} \times \mathrm{N}$ matrix; $\Sigma$ is an $\mathrm{N} \times \mathrm{N}$ diagonal matrix with positive or zero elements representing the singular values of matrix $\mathrm{X} ; \mathrm{V}^{\top}$ is the transpose of $\mathrm{N} \times \mathrm{N}$ matrix. Data matrix $\mathrm{X}$ is designed with time variations as column-wise and 
spatial variations as row-wise. The columns of matrix $U$ includes the set of EOF (Empirical Orthogonal Function) which describes spatial variations of data. Indeed, columns represent the directions of the greater space energetics variation of the experiment. They are orthogonal to each other and represent the axis of the orthogonal empirical base. They are arranged with the decreasing of the energetics importance. Accordingly, the first column of the $U$ matrix is named EOF1, it is the most energetics direction of the experimentation empirical base. Then, the second column of the matrix $U$ is named EOF2 and is the orthogonal direction, with an energetics perspective just lower than the previous one. That carries on to the EOF $\mathrm{m}$ column. $\Sigma$ is a rectangular and diagonal matrix with $\mathrm{M}$ lines and $\mathrm{N}$ columns. The whole of the diagonal values coincides with the representativeness of the previous "Empirical Orthogonal Function". The first value $\Sigma_{1}$ of the matrix $\Sigma$ is the energetics representativeness of the EOF1. In the same way the second value $\Sigma_{2}\left(\Sigma_{2}<\Sigma_{1}\right)$ is the energetics representativeness of the EOF2 and the calculation goes up to $\Sigma_{\mathrm{m}} \mathrm{V}$ is a square matrix with $\mathrm{N}$ lines and $\mathrm{N}$ columns. Each column is orthogonal to the others. The columns represent the directions of greater temporal energetics variation of the experiment. It moves away from the physical representation of the experimentation.

\subsection{Application to improve the delamination detection}

Both studies were preliminary research to investigate the effectiveness of SVD on the basis of various applications of EOF. While the first EOFs are dedicated to photothermal variations at the surface of the material, rather than EOFs of higher levels allow to detect thermal variations in the thickness. Then, results were applied to a specific case as the detection of the biocolonisation of stone that induced artefacts at the surface of the substrate.

\subsubsection{Theoretical study}

Simulations of flaws detection like delaminations in mural paintings were carried out with IRT analysis in the aim at testing the effectiveness of the SVD decomposition [6]. For this purpose, an experiment was set up with flaws as air strips hidden inside a plaster block. Six defects were spread at a distance of $2 \mathrm{~mm}$ of each other and at different levels in the block. The first flaw was near the surface, the second one at $6 \mathrm{~mm}$ and the last one at 10 $\mathrm{mm}$ deep. The device was assessed by pulsed IRT analysis in the goal to detect flaws by a non-destructive technique. The pulsed IRT consisted in heating the sample for 2 seconds; it is reached by means of flash lamps corresponding to a deposited power of $1500 \mathrm{~W}$. The excitation flux is 1.5 times higher in the lower part than in the upper part in the aim at simulating a variation of absorptivity of the substrate between the both sides. After the heating excitation, the temperature rises that is detected by IRT recording for 200 seconds. The photothermal signature was clearly different in the both sides and the detection of heterogeneities was more obvious in the lower side which had received a higher energy deposit (figure 1). Therefore, the pulsed IRT has shown up the variation of absorptivity at the surface in the both sides rather than detecting the flaws inside the substrate. Accordingly, SVD analysis has been carried out to focus the flaws detection. Post processed images as the very first EOF still displayed a sensitivity to the heterogeneity of the energy deposit but the calculation of the next EOFs was less dependent from EOF2. Finally, EOF 5 showed no variation depending on the energy flux and it detected only flaws in the substrate (figure 2).

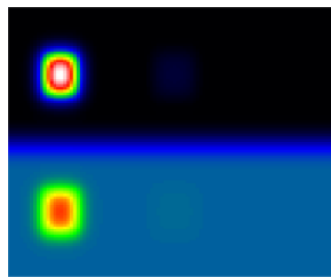

$t=28 s$

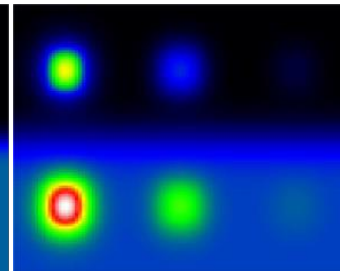

$t=91 s$

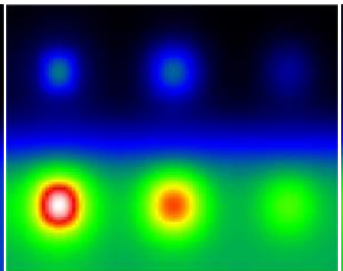

$t=177 \mathrm{~s}$

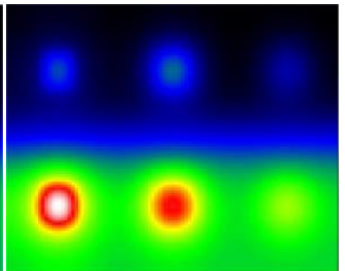

$t=200 s$

Fig. 1. Theoretical thermograms over time (Mouhoubi et al., 2014). 


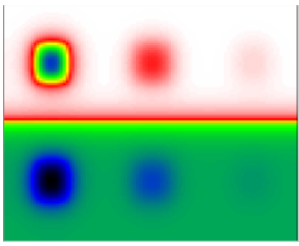

EOF 0

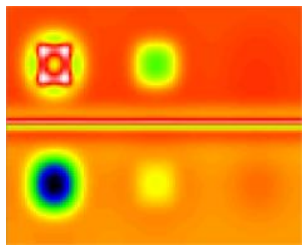

EOF 3

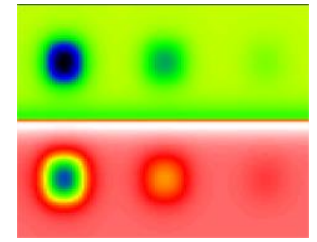

EOF 1

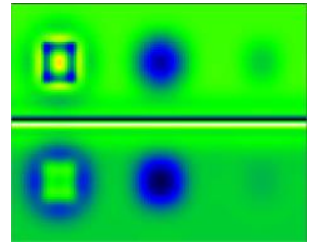

EOF 4

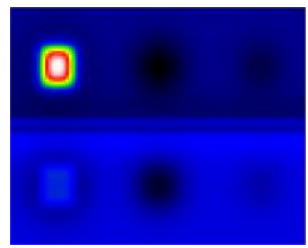

EOF 2

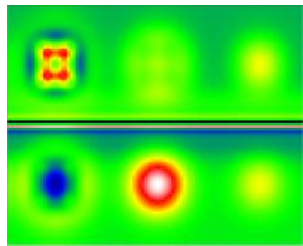

EOF 5

Fig. 2. The first 6 theoretical EOF obtained by SVD decomposition (Mouhoubi et al., 2014).

\subsubsection{Experimental study}

This second study was set up to apply the theoretical one. It was made of a plaster block with twenty colourful square patterns (figure 3). These layer paints displayed the visible range and the radiative properties. Indeed, black colour has a high absorptivity whereas the white one has a high diffusion and silver one has a high reflectivity. The variations of colour properties lead to a space inhomogeneous energy deposit. Thirty-two discs of extruded polystyrene were spread inside the plaster block at $5 \mathrm{~mm}$ depth.

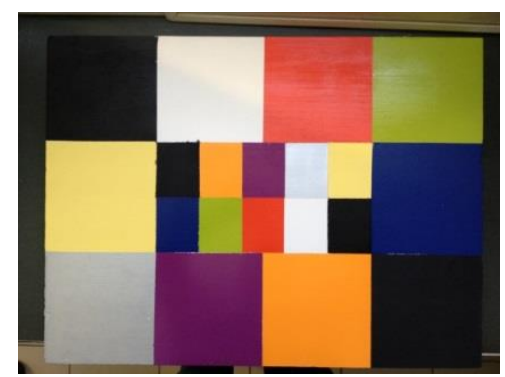

Fig. 3. The studied sample (front face) (Mouhoubi et al., 2014).

The set up was made of a couple of halogen lamps for the energy excitation. An IRT bolometer camera (A20 FLIR) recorded the photothermal signal. The excitation energy was $500 \mathrm{~W}$ as a crenel type for $1 \mathrm{~min}$. The recording time was $3 \mathrm{~min}$ and the acquisition frequency was $1 \mathrm{~Hz}$. Thermograms resulted from the recording at $10 \mathrm{~s}, 60 \mathrm{~s}$ and $120 \mathrm{~s}$, they showed the detection of discs inside the block and of the different patterns that depends on the colour (figure 4). The SVD treatment of thermograms displayed that the first EOF was influenced by the energy deposit at the surface and was subjected to the colour. The next EOF from EOF3 highlighted the discs shape at the expense of the colourful patterns (figure 5) Therefore, the first EOF emphased the superficial variations of energy deposit, then the increasing of EOF detected deeper energy variations. Consequently, SVD decomposition is a useful mathematical analysis to detect flaws at different depths inside a work of art as a painting.

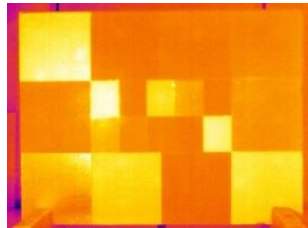

$t=10 s$

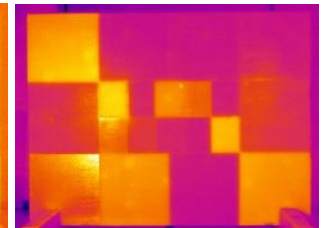

$\mathrm{t}=60 \mathrm{~s}$

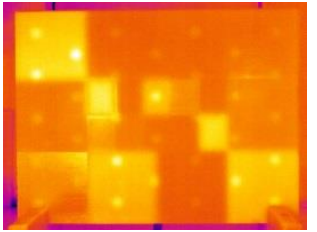

$\mathrm{t}=120 \mathrm{~s}$

Fig. 4. Experimental thermograms recording at $t=10 \mathrm{~s}, t=60 \mathrm{~s}$ and $t=120$ s (Mouhoubi et al., 2014). 


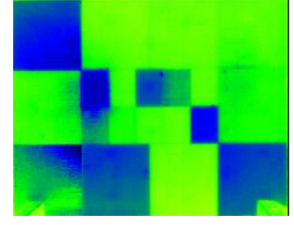

EOFO

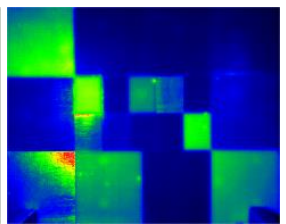

EOF1

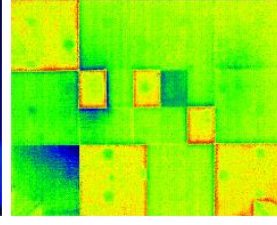

EOF2

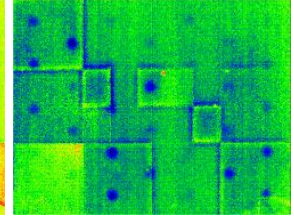

EOF3

Fig. 5. The first 4 experimental EOF calculated from SVD decomposition (Mouhoubi et al., 2014).

\section{Detection of biofilms on stone by IRT}

Because of the great expertise of IRT in Cultural Heritage for many years and benefiting the last promising results, this non-destructive technique was tested to track the pioneer natural colonisation on stone buildings which is not visible to necked eyes as various bacteria and fungi. Such communities are often organised in biofilms to live in drastic conditions by producing extracellular polymeric substances (EPS) that keep water for long periods, maintain the viability of the cells and help access to water vapour in the atmosphere [7]. Biofilms colonise every substrate in every environment but their development time varies mostly with the substrate properties, climatic conditions and exposure. For that reason, porous limestones provide a good environment to the development of biofilms as Courville stone which was used to set up an accelerated biofouling test.

\subsection{Substrate: Courville Stone}

Courville stone has been selected for this experiment because it is one of the stones the most employed from antiquity to nowadays in major buildings and monuments like many Gothic cathedrals in many French regions around Paris. It is dated to the Lutetian age (45 My - tertiary era). It comes from quarries in Rheims surroundings located at $100 \mathrm{~km}$ to the East of Paris. Courville stone is a clear yellow limestone with coarse white shells and foraminifera (milliolids, Orbitolites complanatus and Alveolinidae) [8]. It is a microporous stone with a porosity between 13 and $22 \%$. The last building stone quarry which is now closed from 2005 was at Courville village and provided the stone for the restoration of Rheims cathedral.

\subsection{Outdoor biofouling test}

The test consisted of natural ageing of stone samples in the goal to develop micro-organisms on stone over time. Small samples of Courville stone (dimensions: $2 \times 2 \times 1 \mathrm{~cm}$ ) have been settled on a galvanized steel platform, $1 \mathrm{~m}$ above the ground and $20^{\circ}$ tilted to the SW to limit the stagnation of water and maximize sunshine. It located in outdoor, in the garden of Sacré Coeur secondary school in Rheims city (figure 6). Before setting up the stone samples on the platform, they were sterilized to kill bacteria still developed on the stone surface to start a new natural seeding from the beginning of the test. The experiment started in November 2016 and the first samples have been removed at six months of ageing to be conditioned at room temperature and humidity in order to be investigated in IRT.

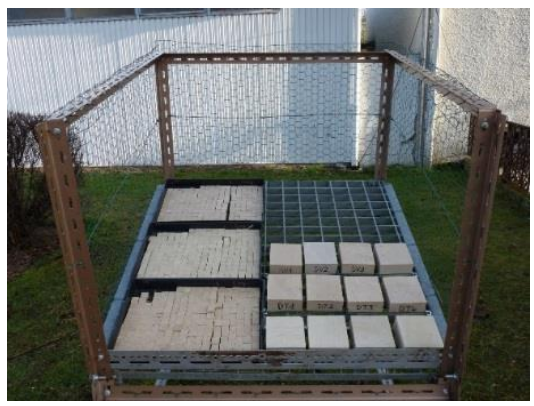

Fig. 6. Platform of stone samples exposure in outdoor. Smaller ones were collected for IRT testing. 


\subsection{IRT procedure}

Infrared thermography is a useful technique extensively applied to many fields including Cultural Heritage during the last two decades. It provides an analysis of surface and subsurface structure and detect artefacts of heat diffusion in many materials as painting, stone, wood, etc. Deterioration processes change the thermal parameters of the material, IRT can reveal inhomogeneities and artefacts of the surface and the subsurface structure.

In that regard, the NDT was investigated to detect early biofilms on stone with pulsed IRT. This technique assesses the thermal response of the sample when a flux of photons, emitted by a short ring flash, excites it for 5 ms. Here the device is $20 \mathrm{~cm}$ diameter and the power of excitation was $3000 \mathrm{~J}$ (figure 7).
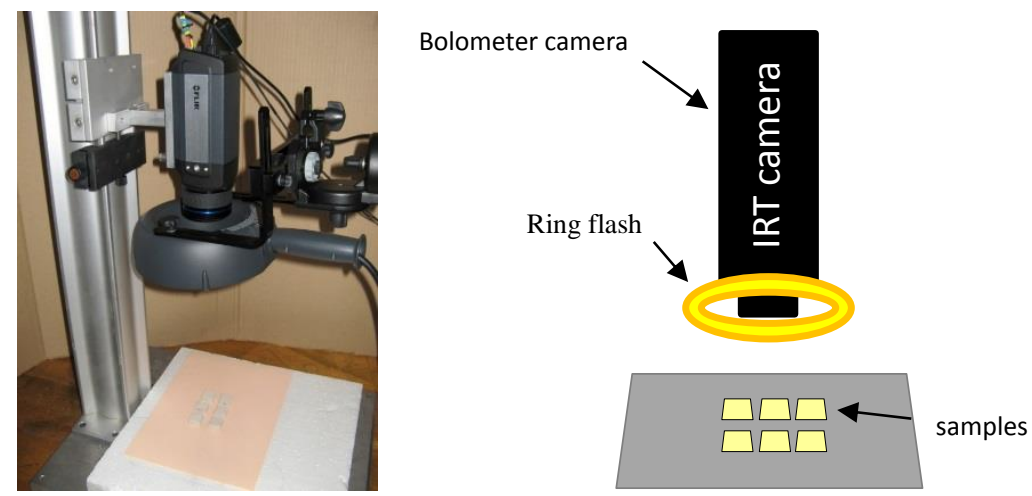

Fig. 7. Device of the laboratory test, IRT is stimulated by a ring flash on stone surfaces. Temperature variations are recorded by a bolometer camera.

Six stone samples were investigated with $2 \times 2 \times 1 \mathrm{~cm}$ dimension (figure 8 ). Three upper samples were sterile stones non-exposed in outdoor and three lower ones were exposed in outdoor for six months then collected for testing. The temperature provided by the flash is considered uniform on the samples area and the heat diffusion takes place along an orthogonal direction to the samples. After the sharp rise, the temperature decreased with time due to the diffusion into the samples. The temperature variations were recorded by a bolometer camera (FLIR SC655) during 5 seconds and the acquisition frequency was equal to 50 Hertz.

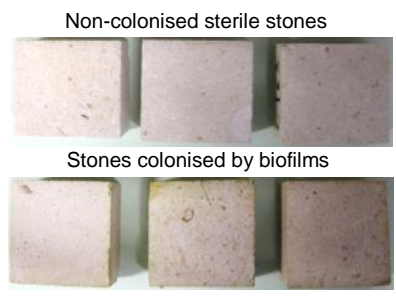

Fig. 8. Samples testing in pulsed IRT. The upper samples are controls without biological colonisation, the lower ones are colonised at 6 months of exposure. Photo in natural light.

\section{Results}

The photothermal response after the excitation of the three colonised stones was similar to control ones. Slight thermal variations were detected and displayed that microfossils were highlighted in pink colour whereas macroporosity was in black colour. Thus the heterogeneities of every stone related to its intrinsic properties were detected by a pulsed IRT but no thermal variations were noticed between colonised samples and controls. The biofilms developed at the stone surface in outdoor were not detected in the raw photothermal films (figure 9). 


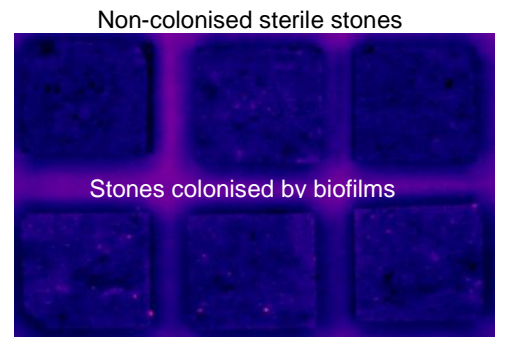

Fig. 9. IRT image after a flash stimulation of non-colonised stones (control) and stones colonised by biofilms.

Nenetheless, SVD decomposition was applied on the thermogram to display variations not detected without treatment yet. As a result, the calculation of "Empirical Orthogonal Function" (EOF) showed that the first ones, EOF 0 to EOF 2, highlighted variations between samples colonised by biofilms and controls without biofilm (figure 10). The first EOFs corresponds to the most significant variations of the signal, in the most energetic direction. They are more sensitive to the variations of energy at the surface of the substrate than to its intrinsic heterogeneities. Therefore, SVD treatment was a useful tool to detect low thermal variations induced by the development of biofilms at the surface of the stone.

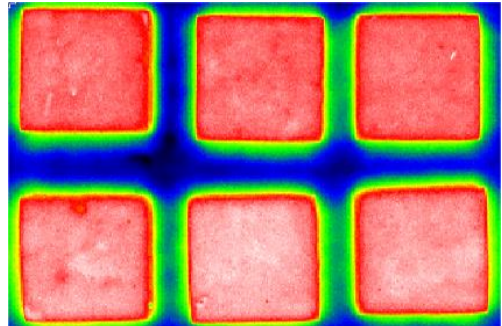

EOF 0

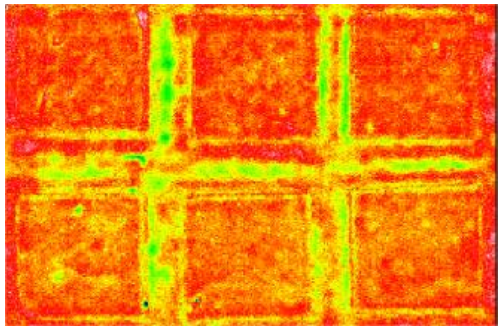

EOF 2

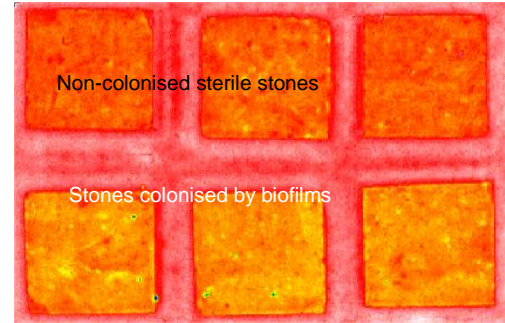

EOF 1

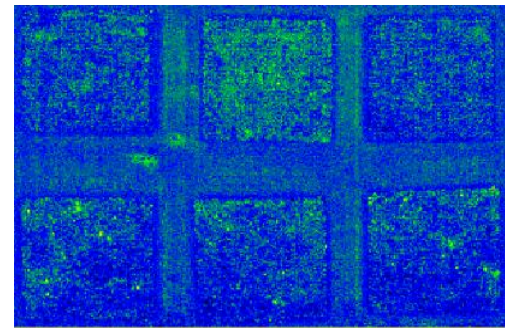

EOF 3

Fig. 10. Comparison between non-colonised stones and colonised stones in IRT treatment as SVD decomposition for EOF 0, EOF 1, EOF 2 and EOF 3.

\section{Conclusion}

In the context to avoid the fouling of stone in the historical monuments by the biological development, the early detection of biocolonisation as bacterial biofilms at the stone surface with IRT allows to react more rapidly and effectively before the greening of the walls. The SVD as post processing of thermograms is usually an efficient mathematical treatment enables a better analysis by avoiding the surface artefacts and helping the detection of flaws in substrate depth. On the opposite, the novelty of our study was to use SVD to detect the biofilms on stones. While the raw thermogram detected the heterogeneities as micro-fossils inside the stone, the first EOF of SVD highlighted biofilms as a variation of energy at the stone surface. 


\section{REFERENCES}

[1] C.M. Grossi, P. Brimblecombe, R.M. Esbert, F.J. Alonso, Color changes in architectural limestones from pollution and cleaning, Color Res. Appl. 32 (2007) 320-331. doi:10.1002/col.20322.

[2] F. Borderie, N. Tête, D. Cailhol, L. Alaoui-Sehmer, F. Bousta, D. Rieffel, L. Aleya, B. Alaoui-Sossé, Factors driving epilithic algal colonization in show caves and new insights into combating biofilm development with UVC treatments, Sci. Total Environ. 484 (2014) 43-52. doi:10.1016/j.scitotenv.2014.03.043.

[3] S. Eyssautier-Chuine, M. Gommeaux, C. Moreau, C. Thomachot-Schneider, G. Fronteau, J. Pleck, B. Kartheuser, Assessment of new protective treatments for porous limestone combining water-repellency and anti-colonization properties, Q. J. Eng. Geol. Hydrogeol. 47 (2014) 177-187. doi:10.1144/qjegh2013-026.

[4] D. Gavrilov, R.G. Maev, D.P. Almond, A review of imaging methods in analysis of works of art: Thermographic imaging method in art analysis, Can. J. Phys. 92 (2014) 341-364. doi:10.1139/cjp-2013-0128.

[5] K. Mouhoubi, J.-L. Bodnar, J.L. Nicolas, E. Perrin, V. Vrabie, V. Detalle, J.M. Vallet, T. Duvaut, Non destructive testing of works of art by stimulated infra-red thermography: Study of the contribution of SVD decomposition to the reduction of the optical effects induced by the pictorial layer, in: Quant. Infrared Thermogr. Conf. QIRT 14, Bordeaux (France), 2014.

[6] N. Rajic, Principal component thermography for flaw contrast enhancement and flaw depth characterisation in composite structures, Compos. Struct. 58 (2002) 521-528. doi:10.1016/S0263-8223(02)00161-7.

[7] A.A. Gorbushina, Life on the rocks, Environ. Microbiol. 9 (2007) 1613-1631. doi:10.1111/j.14622920.2007.01301.x.

[8] G. Fronteau, Comportements télogénétiques des principaux calcaires de Champagne-Ardenne: en relation avec leur facies de dépôt et leur sequençage diagénétique, Ph.D thesis, University of Rheims ChampagneArdenne, 2000. https://www.theses.fr/2000REIMS011 (accessed February 19, 2018). 